\title{
The Role of Flux Cancellation in Eruptions from Bipolar ARs
}

\author{
S. L. Yardley ${ }^{1,2}$ (1) , L. M. Green ${ }^{2}$ (1), L. van Driel-Gesztelyi ${ }^{2,3,4}$, D. R. Williams ${ }^{5}$, and D. H. Mackay ${ }^{1}$ (1) \\ ${ }^{1}$ University of St Andrews, School of Mathematics and Statistics, North Haugh, St Andrews, Fife KY16 9SS, UK \\ ${ }^{2}$ University College London, Mullard Space Science Laboratory, Holmbury St. Mary, Dorking, Surrey RH5 6NT, UK \\ ${ }^{3}$ Observatoire de Paris, LESIA, UMR 8109 (CNRS), F-92195 Meudon-Principal Cedex, France \\ ${ }^{4}$ Konkoly Observatory of the Hungarian Academy of Sciences, Budapest, Hungary \\ ${ }^{5}$ ESA European Space Astronomy Centre, E-28692 Villanueva De La Cañada, Madrid, Spain \\ Received 2017 October 24; revised 2018 August 18; accepted 2018 August 29; published 2018 October 5
}

\begin{abstract}
The physical processes or trigger mechanisms that lead to the eruption of coronal mass ejections (CMEs), the largest eruptive phenomenon in the heliosphere, are still undetermined. Low-altitude magnetic reconnection associated with flux cancellation appears to play an important role in CME occurrence as it can form an eruptive configuration and reduce the magnetic flux that contributes to the overlying, stabilizing field. We conduct the first comprehensive study of 20 small bipolar ARs (ARs) in order to probe the role of flux cancellation as an eruption trigger mechanism. We categorize eruptions from the bipolar regions into three types related to location, and find that the type of eruption produced depends on the evolutionary stage of the AR. In addition, we find that ARs that form eruptive structures by flux cancellation (low-altitude reconnection) had, on average, lower flux cancellation rates than the AR sample as a whole. Therefore, while flux cancellation plays a key role, by itself it is insufficient for the production of an eruption. The results provide supporting evidence that although flux cancellation in a sheared arcade may be able to build an eruptive configuration, a successful eruption depends upon the removal of sufficient overlying and stabilizing field. Convergence of the bipole polarities also appears to be present in regions that produce an eruption. These findings have important implications for understanding the physical processes that occur on our Sun in relation to CMEs and for space weather forecasting.
\end{abstract}

Key words: Sun: activity - Sun: corona - Sun: coronal mass ejections (CMEs) - Sun: evolution - Sun: magnetic field - Sun: photosphere

Supporting material: animation

\section{Introduction}

Coronal mass ejections (CMEs) are the most energetic phemonena in the solar system, involving around $10^{32}$ ergs of energy in the form of electromagnetic, kinetic, thermal, nonthermal, and gravitational potential energy. The energy is ultimately derived from the coronal magnetic field, where it is stored in the form of electric currents (Forbes 2000). However, the exact evolution of the coronal magnetic field, and the physical processes involved in CMEs, are still subjects of study. CMEs are also of interest because they can drive intense geomagnetic storms (Gosling 1993). These storms are able to create hazardous space weather conditions at Earth, leading to disruptions of our technological systems, and causing significant socioeconomic impact (for a review see Eastwood et al. 2017). Understanding the conditions in which CMEs are created is therefore of importance for a physical understanding of our Sun, as well as for space weather forecasting.

The occurrence of CMEs involves an energy storage-andrelease process, and their formation is often discussed as having two phases; a trigger and a driver. The trigger refers to the physical process(es) that brings the magnetic field to the point of an eruption, whereas the driver is responsible for the sudden expansion and upward acceleration of the erupting volume. The driving mechanism appears to be limited to either magnetic reconnection taking place in a vertical current sheet below the eruptive structure (Moore et al. 2001) or the Lorentz force acting on a flux rope (Forbes \& Isenberg 1991; Török \& Kliem 2005; Kliem \& Török 2006; Mackay \& van Ballegooijen 2006a, 2006b; Kliem et al. 2014). Possible trigger mechanisms, however, appear to be wide-ranging and include, for example, sunspot rotation, flux emergence, and photospheric flows. (See Green et al. 2018 for an overview of CME trigger and driver processes.) Our efforts to understand (and forecast) CMEs are severely impeded by a lack of knowledge of the relative importance of these trigger mechanisms.

In this study we focus on another particular CME trigger known as flux cancellation. In the flux cancellation process small-scale opposite polarity magnetic fragments are seen to converge, collide, and disappear along the polarity inversion line (PIL) that separates regions of positive and negative field in the photosphere (Martin et al. 1985). The disappearance of the two opposite polarity fragments is ultimately the consequence of the fragmentation and dispersion of the magnetic field caused by convective flows and differential rotation. Three scenarios have been proposed to explain the process of flux cancellation (see Zwaan 1987): the emergence of a U-loop (van Driel-Gesztelyi et al. 2000; Bernasconi et al. 2002), the submergence of an $\Omega$-loop below the surface (Harvey et al. 1999; Chae et al. 2004; Yang et al. 2009), or the result of magnetic reconnection taking place at a low height (van Ballegooijen \& Martens 1989). We investigate the third case where flux cancellation due to low-altitude magnetic reconnection is able to gradually transform a sheared arcade field into a flux rope. In this scenario, magnetic reconnection produces two loops: (1) a small loop with a high curvature, which submerges below the photosphere leading to the disappearance of the small bipole; (2) a loop much larger in size-scale that extends into the corona. Ongoing flux cancellation can therefore form a flux rope that is expected to have its underside located in the 
high plasma- $\beta$ environment of the lower solar atmosphere. During the flux cancellation process an amount of flux equal to that which is canceled is available to be built into the flux rope. The actual amount of flux that is built into a flux rope depends on the properties of the region, such as the amount of shear and the length of the PIL along which flux cancellation is occurring. The details of this process are discussed in Green et al. (2011).

The flux cancellation process also has a secondary effect in that it reduces the flux in the region that contributes to the field, overlying and stabilizing the flux rope. If enough flux is transformed from the overlying arcade into the flux rope, a force imbalance can occur leading to a catastrophic loss of equilibrium and a CME (Lin \& Forbes 2000; Bobra et al. 2008). Or, if the active region (AR) evolves to a point where the overlying field decreases rapidly enough with height, the flux rope can become torus unstable (Kliem \& Török 2006). In this way, flux cancellation can be viewed as a CME trigger mechanism, which in itself requires a converging flow, in a sheared field, to bring opposite polarity fragments together. Such a scenario for flux rope formation and eruption due to flux cancellation is well supported by simulations (Amari et al. 2003; Aulanier et al. 2010) and observations (Green et al. 2011; Yardley et al. 2016).

Here we present the first comprehensive study of the eruptive activity in a representative sample of 20 small bipolar ARs in order to probe the role of flux cancellation as a CME trigger. We study the evolution of the photospheric magnetic field to quantify the significance of flux cancellation in building an eruptive magnetic field environment. We investigate at what point in an AR's lifetime eruptions occur.

\section{Data and Methods}

\subsection{AR Selection}

In this study, we focus on eruptions that are produced in bipolar ARs. Bipolar ARs are selected for study due to their low magnetic complexity, which minimizes the number of PILs along which eruptions might originate. ARs were selected using the following criteria. The regions must have two dominant magnetic polarities with no major mixing of the opposite polarities. The ARs must be isolated from other ARs so that flux cancellation occurring along any external PILs is negligible allowing flux cancellation along the internal PIL to be quantified. They must be short-lived regions and form east of central meridian so that their evolution can be tracked across the disk. Finally, the ARs must emerge within $60^{\circ}$ the central meridian due to the decreasing reliability of the magnetic flux measurements with increasing distance from disk center. The above criteria led to the selection of 20 ARs from the Helioseismic and Magnetic Imager (HMI) era, spanning a time period from 2012 March to 2015 November.

It should be noted that the above criteria led to the selection of small ARs, with magnetic flux $\sim 10^{20}-10^{21} \mathrm{Mx}$. Eruptions from these regions may produce relatively subtle signatures in extreme ultraviolet (EUV) data and no observable CME in white light coronagraph data. Due to this, we do not use the term CME in this work. Rather we refer to eruptions that are identified in EUV data. These eruptions may be successful or may not be fully ejected from the Sun, leading to a failed eruption. With such weak events it can be hard to discriminate these two categories but since we are interested in the role of flux cancellation as a CME trigger, we do not focus on whether each event is failed or fully ejective, only whether it was initiated in the first place.

\subsection{Coronal Evolution and Eruptive Activity}

The coronal evolution of each AR is monitored using EUV images produced by the Atmospheric Imaging Assembly (AIA; Lemen et al. 2012) instrument on board the Solar Dynamics Observatory (SDO; Pesnell et al. 2012). The AIA instrument provides full-disk observations with a high spatial resolution and temporal cadence of 1.15 and $12 \mathrm{~s}$, respectively, for three UV-visible and seven EUV bandpasses. In this study we focus on using 171 and $193 \AA$ to analyze the coronal evolution of each AR. The $171 \AA$ passband is dominated by plasma emission at a temperature of around $0.6 \mathrm{MK}$, whereas the temperature response of the $193 \AA$ has two temperature peaks at approximately 1.2 and $20 \mathrm{MK}$.

Each AR is analyzed in order to identify the time and location of eruptions that are produced. Coronal signatures used to indicate the occurrence of an eruption include at least two of the following: the eruption of a filament or EUV loop system, the rapid disappearance of coronal loops and the formation of a post-eruption arcade (flare arcade), flare ribbons and coronal dimmings.

Each eruption is then placed into one of three categories; the aim being to identify which eruptive structures form at a lowaltitude, and can therefore be studied in the context of flux cancellation taking place in the AR, and which are formed by other processes and/or altitudes. The three categories are given the following names: internal PIL events (for the eruption of a low-altitude structure from along the AR's internal PIL); external PIL events (for the eruption of a low-altitude structure along a PIL at the periphery of the AR), high-altitude events (for the eruption of a structure from a high-altitude in the corona and presumably not associated with flux cancellation during the time period studied).

One or more of the following criteria must be met for an eruption to be classified as an internal or external PIL (lowaltitude) event:

1. The low-lying core field of the AR must be opened and reconfigured as new post-eruption (flare) loops form.

2. Any flare ribbons that form must, in the first instance, be immediately next to and run along the PIL.

3. Any dimming regions that form must, in the first instance, be immediately next to the PIL.

One or more of the following criteria must be met for an eruption to be classified as a high-altitude event:

1. The low-lying core field of the AR must not be involved or modified.

2. Any flare ribbons that form must be well separated from the PIL.

3. Any post-eruption (flare) loops that form must be located above the AR core field.

4. Any dimming regions that form must, in the first instance, be remote from the PIL.

One example from each eruption category is shown in Figure 1 and in the associated animation. 

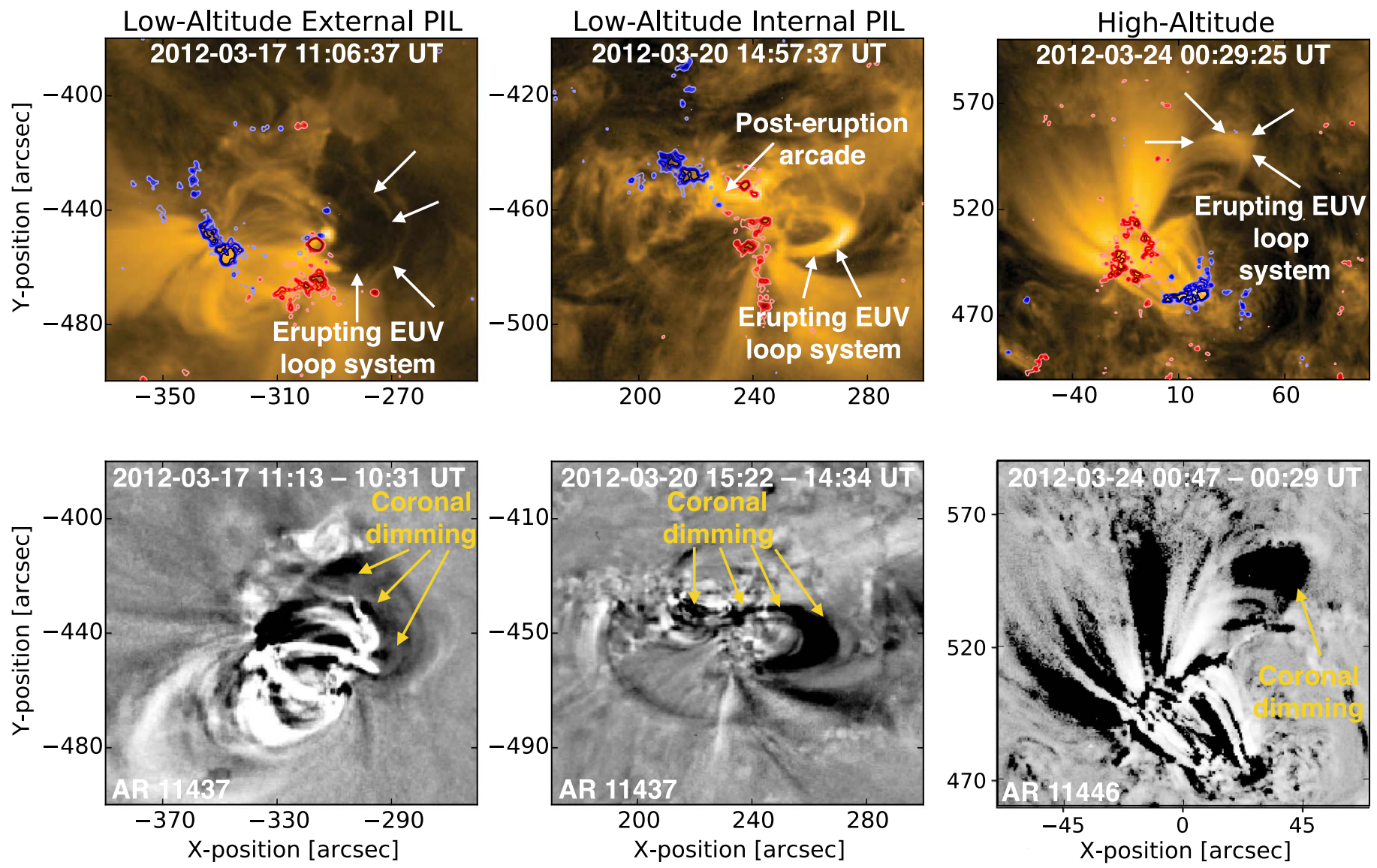

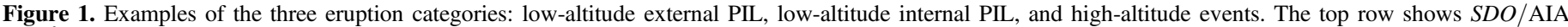

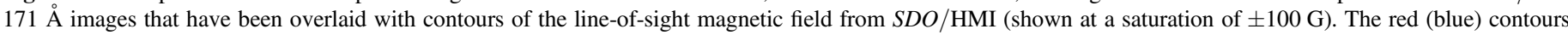

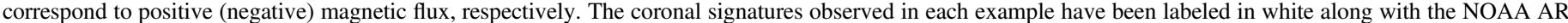

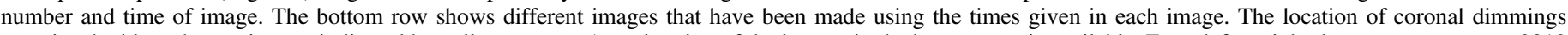

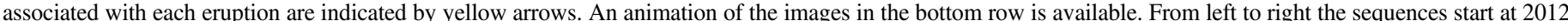

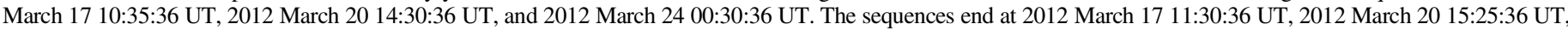
and 2012 March 24 01:25:36 UT, respectively. The video duration is $2 \mathrm{~s}$.

(An animation of this figure is available.)

\subsection{Magnetic Flux Evolution}

The photospheric field evolution of each AR is analyzed using magnetograms obtained by HMI (Schou et al. 2012) on board SDO. The magnetograms used in this study provide information on the line-of-sight component of the magnetic field and are from the $720 \mathrm{~s}$ series (hmi.M_720s) recorded by the vector camera. This camera has a pixel size of $0 .{ }^{\prime \prime} 5$ and a noise level of $10 \mathrm{G}$. The magnetic flux evolution of each AR was calculated by implementing the Solar Tracking of the Evolution of Photospheric Flux (STEF) algorithm (Yardley et al. 2016) on the line-of-sight magnetograms. The cadence of the magnetograms used is 96 minutes.

ARs are manually identified in the full-disk magnetograms and a field of view is assigned as a rectangular box centered on the AR. The radial component of the magnetic field $B_{R}$ is estimated for each pixel in the series of full-disk magnetograms by applying a cosine correction to the longitudinal magnetic field $B_{\mathrm{LOS}}$ using Heliocentric Earth Equatorial coordinates:

$$
B_{\mathrm{R}}=\frac{B_{\mathrm{LOS}}}{\cos \theta \cos \phi},
$$

where $\theta$ and $\phi$ are the helioprojective westward and northward angles, respectively. These angles can be expressed in terms of heliocentric westward and northward coordinates $x, y$

$$
\begin{gathered}
\theta=\arcsin \frac{x}{R \cos \phi}, \\
\phi=\arcsin \frac{y}{R},
\end{gathered}
$$

where $R$ is the radius of the Sun with respect to the observer. The magnetogram containing the radialized field values is then de-rotated to the central meridian passage time of the AR to correct for projection effects using a routine that has been developed in SunPy (SunPy Community et al. 2015).

The flux-weighted central coordinates of the selected field of view are calculated for each time step making it possible to track the AR such that it always remains in the field of view. The pixels that make up the AR are then selected as follows. First, a Gaussian filter is applied to smooth the data with a standard deviation (width) of 7 pixel units. The weighted average of the magnetic flux density of the neighboring pixels must exceed a threshold of $40 \mathrm{G}$. This threshold is set manually and has been tested to give the best results. The largest regions of magnetic flux that form at least $60 \%$ of the selected pixels are identified and retained, whereas the smaller features at large distances are disregarded. This is to ensure that quiet Sun 

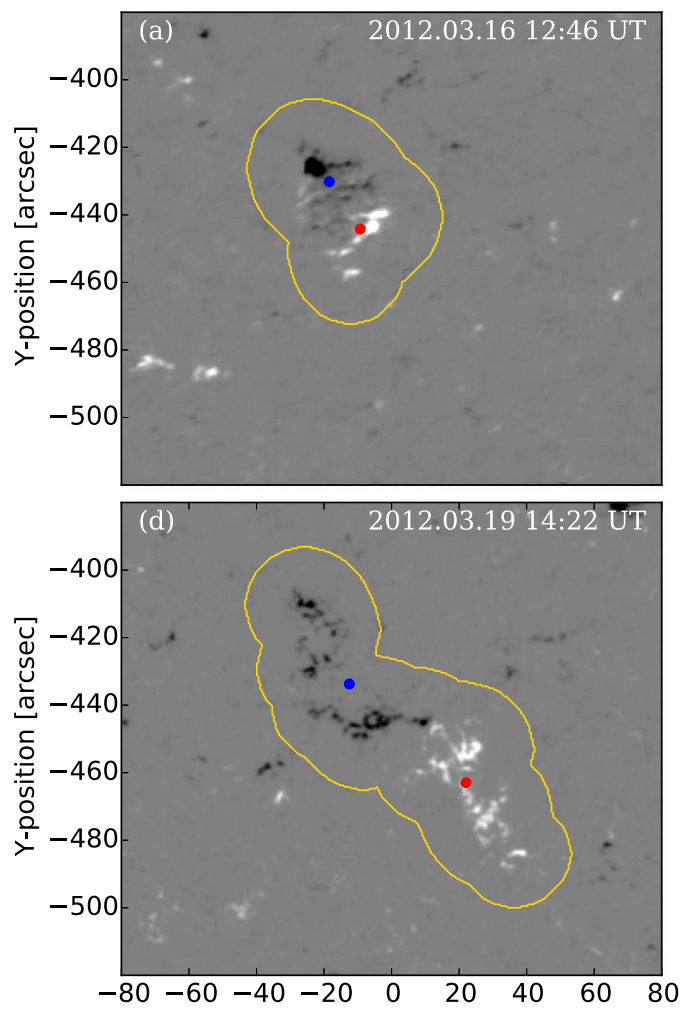
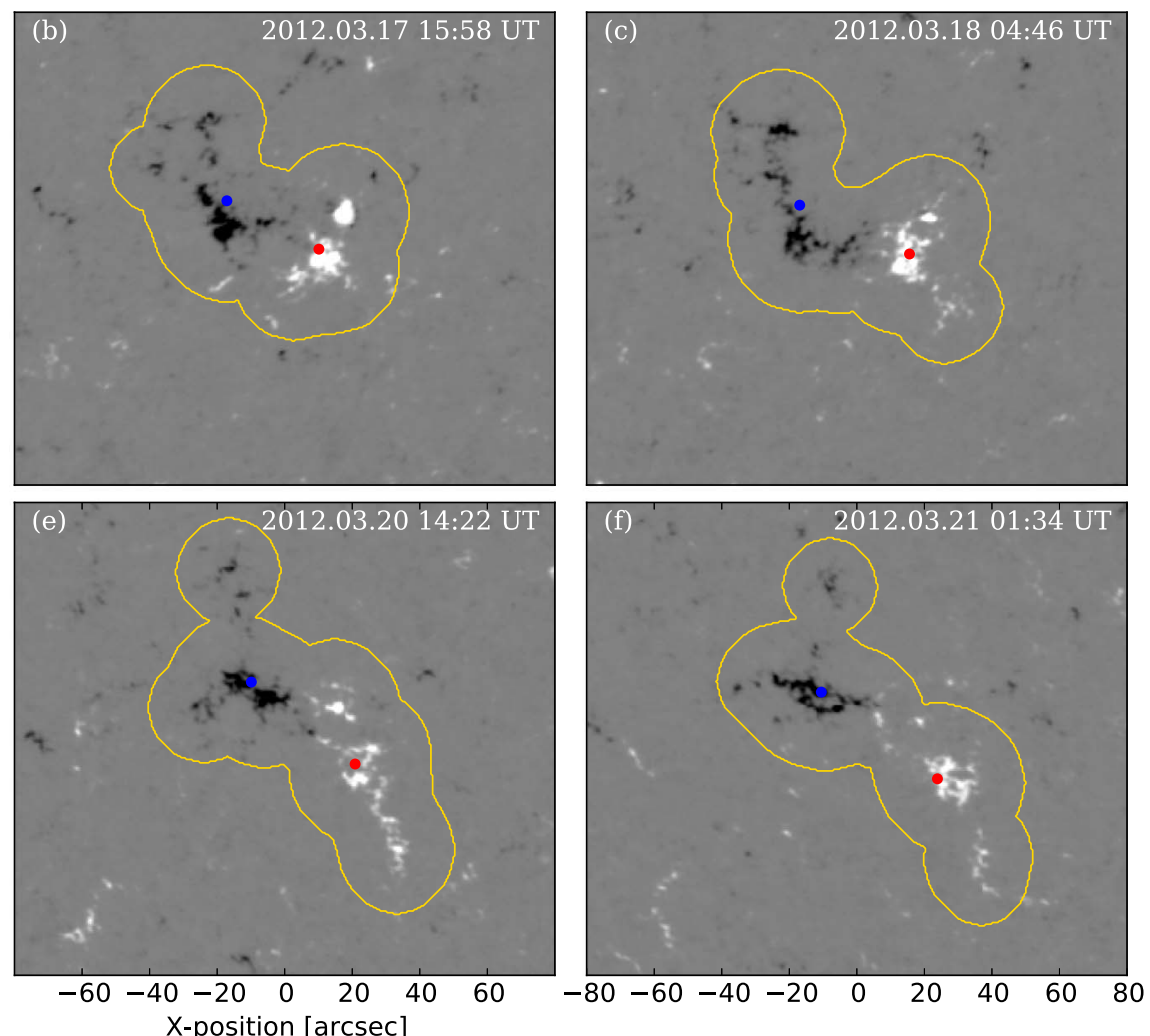

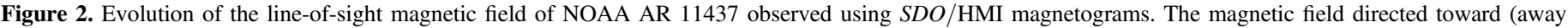

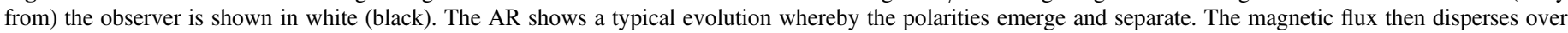

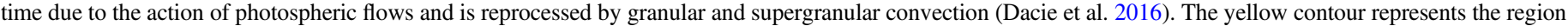
in which the positive, negative, and unsigned flux is calculated. The red (blue) points represent the positive (negative) flux-weighted central coordinates.

magnetic features that are not part of the AR are removed. It is still possible that small-scale magnetic features can enter or exit the boundary, introducing a contribution to or reduction of the magnetic flux measurement. These fluctuations are usually small and an error on the flux measurement is estimated by measuring the magnetic flux of small flux fragments that move in or out of the AR area. This error estimation varies in time as the AR evolves. If the automatic detection fails to successfully select the AR flux at any time step a function is used that allows the user to manually select contours of magnetic flux for the flux calculation. Finally, a dilation is applied so that pixels within approximately $5^{\prime \prime}$ of those selected are also included within the AR area selection. Figure 2 shows the line-of-sight photospheric magnetic field evolution of AR 11437 and the AR area (shown by the yellow contour) identified by the STEF algorithm. Magnetic flux is measured in this area.

The emergence of small bipoles in or close to an AR, or quiet Sun fragments that cancel with flux at the periphery of the $\mathrm{AR}$, can affect the measurement of magnetic flux. In these cases it is not possible to accurately identify and measure the flux cancellation at the internal PIL. To take this into account, if possible, the flux cancellation is not calculated during time periods when external flux cancellation or flux emergence is occurring.

\subsection{Separation Distance of AR Polarities}

To determine whether there is an overall convergence of the two polarities of the bipole, the separation of the positive and negative polarities is calculated. The separation distance is quantified by computing the separation of the flux-weighted central coordinates of the positive and negative bipoles. The flux-weighted central coordinates $f$ are computed as follows

$$
f=\frac{\sum_{i} B_{i} x_{i}}{\sum_{i} B_{i}},
$$

where $B_{i}$ and $x_{i}$ are the magnetic flux density and coordinate corresponding to pixel $i$ of the AR. The flux-weighted central coordinates are computed in both $x$ and $y$. The separation distance $d$ of the polarities is then calculated by

$$
d=\sqrt{\left(x_{1}-x_{2}\right)^{2}+\left(y_{1}-y_{2}\right)^{2}},
$$

where $\left(x_{1}, y_{1}\right)$ and $\left(x_{2}, y_{2}\right)$ are the flux-weighted central coordinates of the positive and negative polarities, respectively.

\section{Results}

\subsection{AR Eruptions}

Of the 20 ARs studied, 13 produced at least one eruption. These 13 ARs produced a total of 24 eruptions during the time period studied. Eight out of these 13 ARs produced lowaltitude events that originated from either the internal or external PIL of the AR. The remaining five of these 13 ARs produced high-altitude events. Table 1 gives the timings of the different types of event as determined by the eruption and rapid disappearance of EUV coronal loops and also the coronal signatures observed during eruption. The majority of the eruptions $(22 / 24)$ that occurred exhibited coronal dimmings, which suggests that these events may have been successful CMEs. However, two of the eruptions are not associated with 
Table 1

This Table Details the Eruptions that Occurred during the Emergence and Decay Phases of Each AR

\begin{tabular}{|c|c|c|c|c|}
\hline $\begin{array}{l}\text { NOAA } \\
\text { AR }\end{array}$ & $\begin{array}{c}\text { Internal } \\
\text { PIL Event Timings (UT) }\end{array}$ & $\begin{array}{c}\text { External } \\
\text { PIL Event Timings (UT) }\end{array}$ & $\begin{array}{c}\text { High-altitude } \\
\text { Event Timings (UT) }\end{array}$ & $\begin{array}{l}\text { Coronal } \\
\text { Signatures }\end{array}$ \\
\hline \multirow[t]{3}{*}{11437} & $\ldots$ & 2012 Mar 17 05:14 & $\ldots$ & $\overline{A, D, L, R}$ \\
\hline & $\ldots$ & 2012 Mar 17 10:53 & $\ldots$ & $A, D, L, R$ \\
\hline & 2012 Mar 20 14:46 & $\ldots$ & $\ldots$ & $\mathrm{A}, \mathrm{D}, \mathrm{L}$ \\
\hline 11446 & $\ldots$ & $\ldots$ & 2012 Mar 24 00:42 & $\mathrm{A}, \mathrm{D}, \mathrm{L}$ \\
\hline 11561 & 2012 Sep 01 23:37 & $\ldots$ & $\ldots$ & $A, D, L, R$ \\
\hline 11680 & 2013 Mar 03 17:27 & $\ldots$ & $\ldots$ & $\mathrm{A}, \mathrm{D}, \mathrm{F}, \mathrm{R}$ \\
\hline \multirow[t]{2}{*}{11808} & $\ldots$ & $\ldots$ & 2013 Jul 30 04:04 & $A, D, L, R$ \\
\hline & $\ldots$ & $\cdots$ & 2013 Jul 31 15:10 & $\mathrm{A}, \mathrm{D}, \mathrm{L}$ \\
\hline \multirow[t]{3}{*}{11881} & $\ldots$ & $\ldots$ & 2013 Oct 24 08:10 (09:12) & A, D, L \\
\hline & $\cdots$ & $\ldots$ & 2013 Oct 27 19:45 & $\mathrm{A}, \mathrm{D}, \mathrm{L}$ \\
\hline & $\cdots$ & $\cdots$ & 2013 Oct 29 02:58 & $\mathrm{A}, \mathrm{D}$ \\
\hline 11886 & $\cdots$ & $\ldots$ & 2013 Oct $2912: 57$ & $\mathrm{D}$ \\
\hline 12086 & $\ldots$ & 2014 Jun 10 14:49 & $\ldots$ & $\mathrm{D}, \mathrm{L}, \mathrm{R}$ \\
\hline \multirow[t]{3}{*}{12119} & $\cdots$ & 2014 Jul 18 10:40 & $\cdots$ & $\mathrm{A}, \mathrm{D}, \mathrm{R}$ \\
\hline & $\cdots$ & 2014 Jul 22 21:02 & $\cdots$ & $\mathrm{D}, \mathrm{L}, \mathrm{R}$ \\
\hline & $\ldots$ & 2014 Jul 23 07:30 (08:12) & $\ldots$ & $A, D, L, R$ \\
\hline \multirow[t]{4}{*}{12229} & $\ldots$ & 2014 Dec 05 03:46 & $\ldots$ & $\mathrm{A}, \mathrm{D}, \mathrm{R}$ \\
\hline & $\cdots$ & 2014 Dec 05 08:12 & $\cdots$ & $\mathrm{A}, \mathrm{D}, \mathrm{R}$ \\
\hline & $\ldots$ & 2014 Dec $0510: 39^{a}$ & $\ldots$ & $\mathrm{R}(\mathrm{C})$ \\
\hline & $\cdots$ & 2014 Dec 05 12:35 & $\ldots$ & $\mathrm{D}, \mathrm{R}$ \\
\hline 12274 & $\cdots$ & 2015 Jan 25 20:00 & $\cdots$ & $\mathrm{L}, \mathrm{R}(\mathrm{C})$ \\
\hline \multirow[t]{2}{*}{12336} & $\cdots$ & $\cdots$ & 2015 May 05 01:29 & $\mathrm{A}, \mathrm{D}, \mathrm{L}$ \\
\hline & $\ldots$ & $\cdots$ & 2015 May 05 09:24 & A, D, L \\
\hline 12382 & 2015 Jul 09 02:29 & $\ldots$ & $\ldots$ & $\mathrm{A}, \mathrm{D}, \mathrm{L}, \mathrm{R}$ \\
\hline
\end{tabular}

Notes. See Table 3 for related AR and flux cancellation information. Column 1 indicates the NOAA AR number. Columns 2, 3, and 4 indicate the timings of internal PIL events, external PIL events and high-altitude events, respectively. Column 4 details the onset time of each eruption as determined by the rapid expansion and eruption of EUV loops. Column 5 gives the coronal signatures observed during an eruption including the eruption of a filament (F), the eruption of an EUV loop system or rapid disappearance of coronal loops (L), post-eruption (flare) arcade or loops (A), flare ribbons (R), and coronal dimming(s) (D). Eruptions that showed no clear coronal dimmings are indicated by $(\mathrm{C})$ in column 5 . These could be confined or failed eruptions.

a The timings of the eruption onset for one internal PIL eruption originating from AR 11680 and one external PIL eruption from AR 12229 were determined by the onset of a filament eruption. One eruption that originated from AR 11881 and one from AR 12119 could also be observed in LASCO/C2. The timings for the LASCO/C2 observations are given in brackets after the timings of the eruption onset.

coronal dimmings and are therefore assumed to be confined or failed eruptions.

It is notable that, during the time period studied, the ARs that produced eruptions produced either internal and/or external PIL events (which originate at a low-altitude) or high-altitude events. No ARs produced both low-altitude and high-altitude eruptions. Overall, eruptions occurred in both the emergence and decay phases of the ARs, however the category of event produced depended on the region's evolutionary stage. Table 2 and Figure 3 show that there is a tendency for external PIL events to occur during an AR's emergence phase and for internal PIL events to form during the decay phase. In contrast to this, the eruption of a high-altitude structure occurs roughly evenly across the emergence and decay phases. The highaltitude events make up only $38 \%$ of the eruptions originating in the ARs.

\subsection{Flux Cancellation}

The flux cancellation rate and total flux canceled in each AR is determined from the reduction in the total unsigned magnetic flux with time (see Figure 4(a)). That is, during the decay phase of each AR. Therefore, the results presented here only refer to the decay phase of the ARs.

Flux cancellation, at either an internal or external PIL, was observed to occur in all but one AR (AR 11867). Table 3
Table 2

This Table Details when Internal PIL, External PIL and High-altitude Eruptions Occur in Relation to the Evolutionary Phase of Their Source AR

\begin{tabular}{lcc}
\hline \hline & $\begin{array}{c}\text { Emergence } \\
\text { Phase }\end{array}$ & $\begin{array}{c}\text { Decay } \\
\text { Phase }\end{array}$ \\
\hline External PIL & 8 & 3 \\
Internal PIL & 0 & 4 \\
High-altitude & 5 & 4 \\
\hline
\end{tabular}

summarizes the flux cancellation rates (column 7) and total amount canceled (column 8). The average flux cancellation rate for all 20 ARs (including AR 11867 that exhibited no cancellation) is $0.84 \times 10^{19} \mathrm{Mx} \mathrm{h}^{-1}$. This compares to an average flux cancellation rate of $0.68 \times 10^{19} \mathrm{Mx} \mathrm{h}^{-1}$ for regions that produce internal and external PIL events, $1.10 \times 10^{19} \mathrm{Mx} \mathrm{h}^{-1}$ for regions that produce high-altitude events, and $0.83 \times 10^{19} \mathrm{Mx} \mathrm{h}^{-1}$ for regions that produce no eruptions at all. Therefore, ARs that do not produce eruptions have a flux cancellation rate close to the average value across all regions, internal and external PIL event ARs have a flux cancellation rate less than average, and high-altitude event ARs have a flux cancellation rate above the average value.

Although flux cancellation can play an important role in the creation of a sheared or twisted pre-eruptive structure 


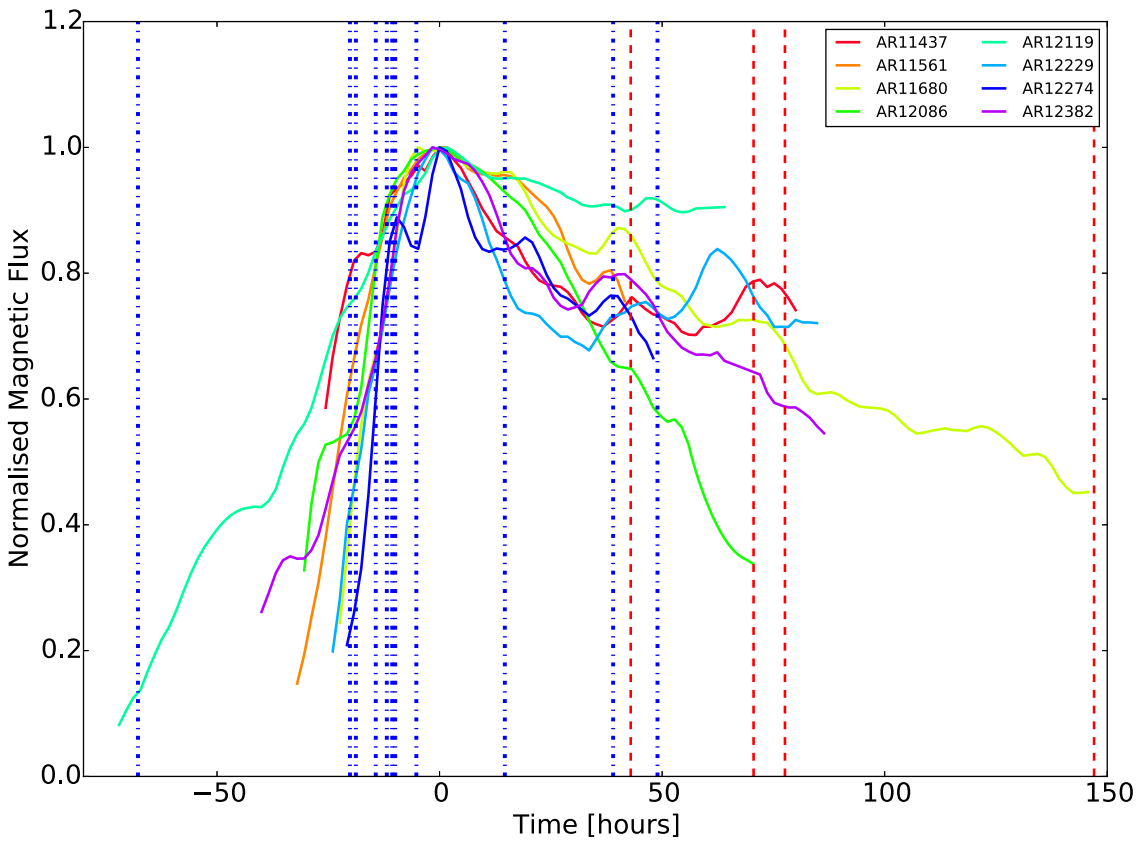

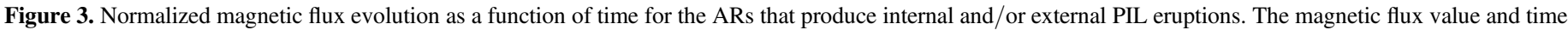

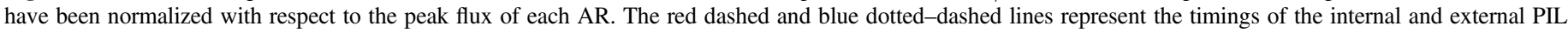
eruptions, respectively.

(van Ballegooijen \& Martens 1989), what is important for the occurrence of an eruption is the force balance between this structure and the overlying arcade field. To investigate this, the total amount of flux canceled must be considered, as this quantity represents the amount of flux that could have been built into a flux rope. This value can then be compared to the amount of flux that remains in the AR as field overlying the rope. Therefore, we now pay particular attention to the four ARs that produce eruptions from their internal PIL, in order to probe how much flux may have been built into the pre-eruptive structure in relation to that remaining as the AR arcade field. We compare these ARs to those that produce high-altitude eruptions. These two groups exhibit a similar amount of total flux canceled at the internal PIL over the time period studied, despite their differing flux cancellation rates. They therefore provide an interesting data set to compare and contrast regions where flux cancellation apparently acts as an eruption trigger (through the creation and eruption of a low-altitude structure) and a group of regions where it does not.

The flux cancellation measurements for the internal PIL event regions and high-altitude event regions are shown in Table 4. The average total flux canceled for the internal PIL event regions and for high-altitude event regions is $4.8 \times 10^{20} \mathrm{Mx}$ and $5.3 \times 10^{20} \mathrm{Mx}$, respectively. The total amount of flux canceled as a quantity and a percentage of the ARs peak flux value can be seen in the third and fourth columns of Table 4 . The percentage values range from $28 \%$ to $49 \%$ for the low-altitude internal PIL events, which is higher than the average value of $24 \%$ of high-altitude event ARs.

Now we compare the amount of total flux canceled (available to be built into the flux rope) to the overlying (and stabilizing) magnetic field that is left in the ARs that produce internal PIL eruptions and high-altitude eruptions. For AR 11561, a corrected flux cancellation value was used to account for the fact that the flux cancellation measurement could not be made during the entire time that cancellation was observed to occur. This is due to the emergence of a bipole to the south of the AR, which cannot be removed from the magnetic flux measurement and therefore masks the flux cancellation taking place at the internal PIL. We find that the ratio of flux canceled, compared to that which remains as overlying field for ARs that produce internal PIL events (at the time of eruption), lies between 1:0.03 and 1:1.57 (see column 5 of Table 4). Here we note that the ratio of 1:0.03 for AR 11437 suggests that practically no overlying field remains, indicating that the assumption that flux cancellation injects an equal amount of flux into the rope may not apply. This compares to ratios between 1:0.94 and 1:3.42 for regions that produce highaltitude eruptions. Therefore, on average, high-altitude event regions had a relatively high value of flux overlying the PIL along which flux cancellation was occurring.

\subsection{Configuration and Motion of AR Polarities}

The orientation of the bipole with respect to the PIL provides information on the level of nonpotentiality (or shear) of the magnetic field. A sheared field is an essential component of the flux rope formation model that is relevant to this study (van Ballegooijen \& Martens 1989). We use the observational proxy of sheared loops to determine the nonpotentiality of the magnetic field of the bipole. The shear angle was measured for each AR at the time of peak magnetic flux (see column 11 of Table 3). The magnetic shear angle is defined as the angle between the normal to the line that connects the flux-weighted central coordinates and the PIL, where the clockwise direction corresponds to a positive shear angle. Nineteen out of the 20 ARs studied showed that flux cancellation and the amount of shear in these 19 regions ranges from $1^{\circ}$ to $40^{\circ}$. We found that, on average, the shear angle of the ARs that produced internal PIL events is significantly higher than the regions that produced high-altitude events. On average, the shear angle for regions that produced internal PIL events and those that 

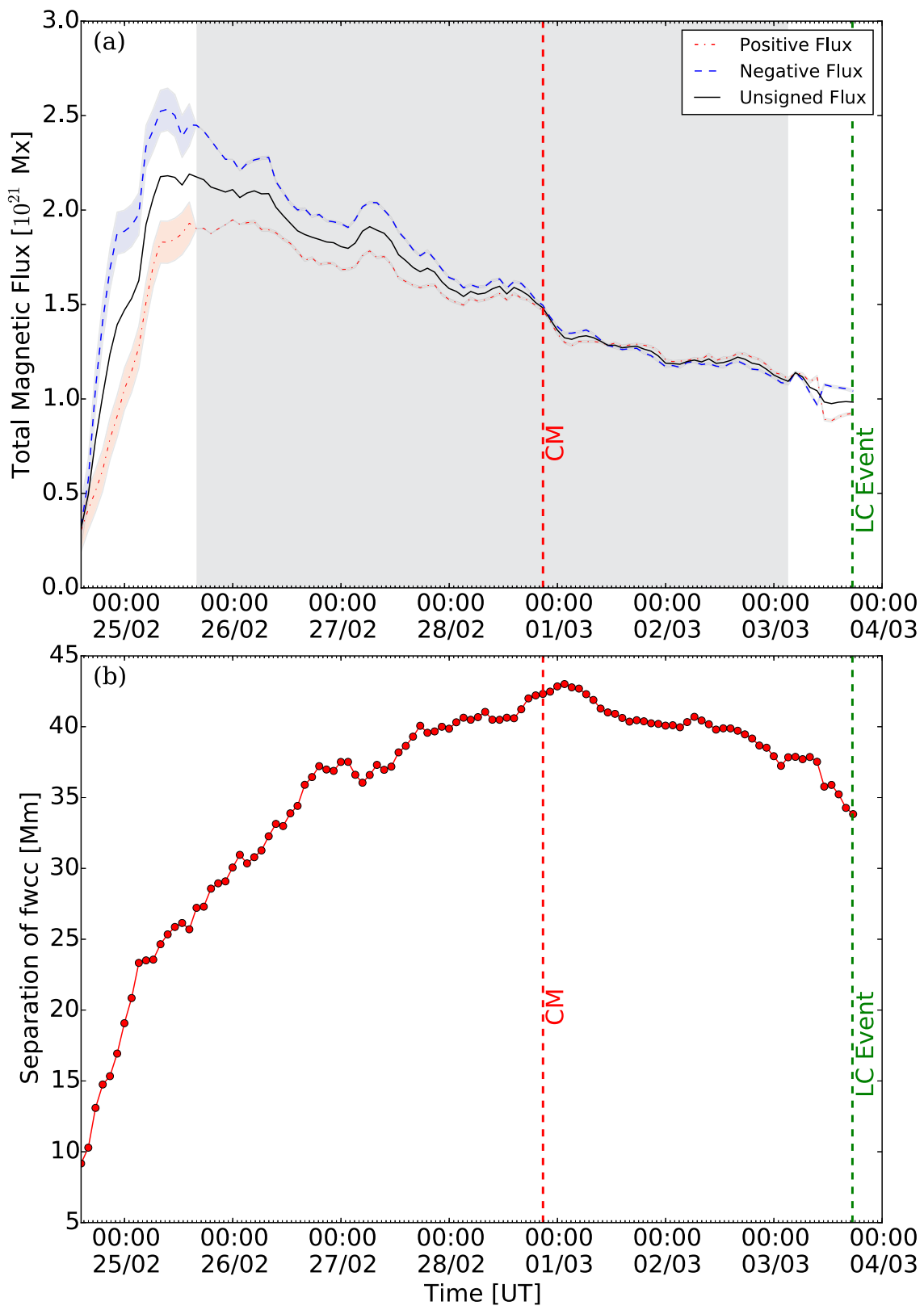

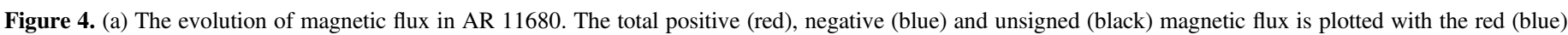

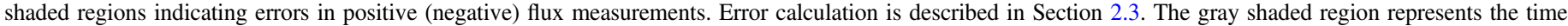

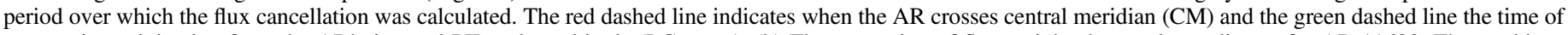

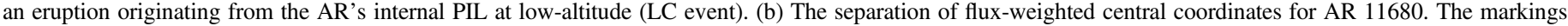
$\mathrm{CM}$ and LC E have the same meaning as in (a).

produced high-altitude events was found to be $28^{\circ}$ and $14^{\circ}$, respectively.

Motion toward the PIL is also an essential component of the model of van Ballegooijen \& Martens (1989) as small fragments of the bipole's magnetic field that break away from the main concentrations converge, collide, and cancel. In addition, an overall convergence of the polarities leads to an inflation of the field that may in turn affect the stability of any flux rope that has formed. Therefore, bipole convergence may also be investigated as a stability proxy. The separation of the AR polarities over time was calculated using the flux-weighted central coordinates of the negative and positive flux regions (see Figure 4(b)). Overall, $75 \%$ of the ARs that produce internal PIL events showed a combination of bipole convergence, shear, and flux cancellation. This is much higher than the subset of ARs that do not produce internal PIL events (34\%). This subset includes regions that produce no eruptions, external PIL eruptions, and high-altitude eruptions.

\section{Discussion}

In this study, we investigate the role of flux cancellation as an eruption trigger in a survey of 20 isolated and small bipolar ARs. Nineteen ARs exhibit flux cancellation, the amount of which was quantified from the reduction in the total unsigned magnetic flux with time. This approach is based on the assumption that flux cancellation is the only process by which AR flux is removed from the photosphere on the timescale of a few days. Other mechanisms of removing flux from an AR 
Table 3

Flux Cancellation of the 20 ARs in This Study

\begin{tabular}{|c|c|c|c|c|c|c|c|c|c|c|c|}
\hline $\begin{array}{l}\text { NOAA } \\
\text { AR } \\
\text { No. }\end{array}$ & $\begin{array}{c}\text { Heliographic } \\
\text { Coordinates } \\
(\theta, \phi)\end{array}$ & $\begin{array}{l}\text { Emergence } \\
\text { Time } \\
\text { (UT) }\end{array}$ & $\begin{array}{l}\text { Peak Flux } \\
\text { Time } \\
\text { (UT) }\end{array}$ & $\begin{array}{l}\text { Flux Cancellation } \\
\text { Start Time } \\
\text { (UT) }\end{array}$ & $\begin{array}{l}\text { Flux Cancellation } \\
\text { End Time } \\
\text { (UT) }\end{array}$ & $\begin{array}{c}\text { Flux Cancellation } \\
\text { Rate } \\
\left(10^{19} \mathrm{Mx} \mathrm{h}^{-1}\right)\end{array}$ & $\begin{array}{l}\text { Total Flux } \\
\text { Canceled } \\
\left(10^{21} \mathrm{Mx}\right)\end{array}$ & $\begin{array}{c}\text { Percentage of } \\
\text { Peak AR Flux } \\
\text { Canceled (\%) }\end{array}$ & $\begin{array}{c}\text { FR Versus } \\
\text { Overlying } \\
\text { AR Ratio }\end{array}$ & $\begin{array}{c}\text { Shear } \\
\text { Angle } \\
\left({ }^{\circ}\right)\end{array}$ & $\begin{array}{c}\text { AR } \\
\text { Bipole } \\
\text { Convergence }\end{array}$ \\
\hline 11437 & S29 E33 & 2012 Mar 16 12:46 & 2012 Mar 17 15:58 & 2012 Mar 17 15:58 & 2012 Mar $2101: 34$ & 0.43 & 0.17 & 31 & $1: 1.29$ & 31 & $\mathrm{Y}$ \\
\hline 11446 & N31 E20 & 2012 Mar 22 15:58 & 2012 Mar 24 15:58 & 2012 Mar 25 11:10 & 2012 Mar 26 03:10 & 1.50 & 0.24 & 24 & $1: 2.26$ & 6 & Y \\
\hline 11480 & S14 E26 & 2012 May 09 11:10 & 2012 May 11 04:46 & 2012 May 11 11:10 & 2012 May 13 23:58 & 0.29 & 0.17 & 25 & $1: 2.38$ & 40 & $\mathrm{~N}$ \\
\hline 11561 & S18 E34 & 2012 Aug 29 19:10 & 2012 Aug 31 04:46 & 2012 Aug 31 04:46 & 2012 Sep 01 14:22 & 0.99 & 0.33 & 28 & $1: 2.55$ & 36 & $\mathrm{Y}$ \\
\hline 11680 & S25 E52 & 2013 Feb 24 14:22 & 2013 Feb 25 14:22 & 2013 Feb 25 15:58 & 2013 Mar 03 03:10 & 0.82 & 1.08 & 50 & $1: 0.03$ & 28 & $\mathrm{Y}$ \\
\hline 11808 & N12 E66 & 2013 Jul 29 01:34 & 2013 Jul 30 01:34 & 2013 Jul 30 19:10 & 2013 Aug 01 11:10 & 2.13 & 0.85 & 29 & $1: 2.57$ & 18 & $\mathrm{~N}$ \\
\hline 11813 & S19 E22 & 2013 Aug 06 01:34 & 2013 Aug 08 17:34 & 2013 Aug 08 17:34 & 2013 Aug 11 23:58 & 1.05 & 0.83 & 31 & $1: 1.19$ & 37 & $\mathrm{Y}$ \\
\hline 11867 & N17 E05 & 2013 Oct 11 07:58 & 2013 Oct 13 15:58 & $\ldots$ & $\cdots$ & $\ldots$ & $\ldots$ & $\cdots$ & $\ldots$ & $\ldots$ & $\cdots$ \\
\hline 11881 & S25 E52 & 2013 Oct 24 01:34 & 2013 Oct 26 15:58 & 2013 Oct 26 15:58 & 2013 Oct 28 14:22 & 1.04 & 0.49 & 34 & $1: 0.94$ & 25 & $\mathrm{~N}$ \\
\hline 11886 & N10 E14 & 2013 Oct 28 09:34 & 2013 Oct 30 17:34 & 2013 Oct 30 17:34 & 2013 Dec 01 04:46 & 0.15 & 0.54 & 24 & $1: 2.26$ & 23 & $\mathrm{Y}$ \\
\hline 12086 & N03 E49 & 2014 Jun 08 15:58 & 2014 Jun 09 23:58 & 2014 Jun 10 15:58 & 2014 Jun 11 15:58 & 1.00 & 0.24 & 30 & $1: 1.67$ & 7 & $\mathrm{Y}$ \\
\hline 12119 & S26 E38 & 2014 Jul 18 04:46 & 2014 Jul 21 06:22 & 2014 Jul 21 06:22 & 2014 Jul 23 01:34 & 0.66 & 0.28 & 11 & $1: 7.05$ & 1 & $\mathrm{Y}$ \\
\hline 12168 & N10 E08 & 2014 Sep 16 12:46 & 2014 Sep 18 11:10 & 2014 Sep 19 20:46 & 2014 Sep 22 14:22 & 0.87 & 0.77 & 37 & $1: 0.79$ & 37 & $\mathrm{Y}$ \\
\hline 12229 & S23 E50 & 2014 Dec 04 20:46 & 2014 Dec 05 22:22 & 2014 Dec 06 04:46 & 2014 Dec 07 09:34 & 0.83 & 0.24 & 32 & $1: 1.3$ & 34 & $\mathrm{Y}$ \\
\hline 12273 & N02 E21 & 2015 Jan 15 15:58 & 2015 Jan 27 19:10 & 2015 Jan 27 19:10 & 2015 Jan 29 03:10 & 0.88 & 0.28 & 10 & $1: 7.74$ & 34 & $\mathrm{Y}$ \\
\hline 12274 & N03 E09 & 2015 Jan 25 17:34 & 2015 Jan 26 15:58 & 2015 Jan 26 15:58 & 2015 Jan 15 01:34 & 0.34 & 0.12 & 28 & $1: 1.41$ & 23 & $\mathrm{~N}$ \\
\hline 12336 & N17 E49 & 2015 May 01 14:22 & 2015 May 05 20:46 & 2015 May 05 20:46 & 2015 May 08 23:58 & 0.70 & 0.53 & 18 & $1: 3.42$ & 1 & $\mathrm{Y}$ \\
\hline 12382 & S08 E29 & $2015 \mathrm{Jul} 04$ 03:10 & 2015 Jul 05 20:46 & $2015 \mathrm{Jul} 05$ 20:46 & $2015 \mathrm{Jul} 09$ 01:34 & 3.50 & 0.27 & 42 & $1: 0.32$ & 19 & $\mathrm{~N}$ \\
\hline 12453 & N04 E29 & 2015 Nov 12 07:58 & 2015 Nov 15 15:58 & 2015 Nov 15 15:58 & 2015 Nov 16 23:58 & 1.58 & 0.51 & 30 & $1: 1.35$ & 32 & Y \\
\hline 12455 & N14 E61 & 2015 Nov 13 04:46 & 2015 Nov 16 03:10 & 2015 Nov 16 06:22 & 2015 Nov 18 01:34 & 1.16 & 0.50 & 35 & $1: 0.84$ & 54 & $\mathrm{Y}$ \\
\hline
\end{tabular}

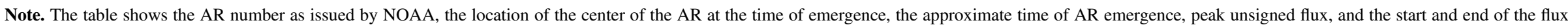

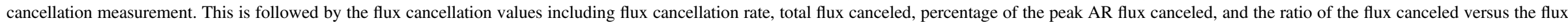

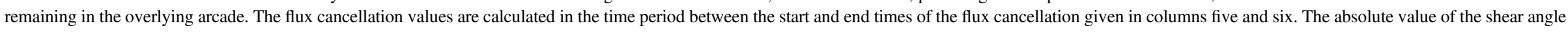
of the AR at the time of peak flux is also given, and whether convergence of the AR bipole is observed. 
Table 4

Flux Cancellation Values for the ARs that Produced Low-altitude Eruptions Originating from the Internal PIL (Top Section) and the ARs that Produced High-altitude Eruptions (Bottom Section)

\begin{tabular}{lcccc}
\hline \hline NOAA & $\begin{array}{c}\text { Flux } \\
\text { Cancellation } \\
\text { Rate } \\
\left(10^{19} \mathrm{Mx} \mathrm{h}^{-1}\right)\end{array}$ & $\begin{array}{c}\text { Total Flux } \\
\text { Canceled } \\
\left(10^{21} \mathrm{Mx}\right)\end{array}$ & $\begin{array}{c}\text { Total } \\
\text { Percentage of } \\
\text { peak AR } \\
\text { flux (\%) }\end{array}$ & $\begin{array}{c}\text { FR Versus } \\
\text { Overlying } \\
\text { Arcade } \\
\text { Ratio }\end{array}$ \\
\hline 11437 & 0.43 & 0.17 & 31 & $1: 1.29$ \\
$11561^{\mathrm{a}}$ & 0.99 & 0.42 & 28 & $1: 1.57$ \\
11680 & 0.82 & 1.08 & 49 & $1: 0.03$ \\
12382 & 0.35 & 0.27 & 43 & $1: 0.32$ \\
\hline 11446 & 1.50 & 0.24 & 23 & $1: 2.26$ \\
11808 & 2.13 & 0.85 & 22 & $1: 2.57$ \\
11881 & 1.04 & 0.49 & 34 & $1: 0.94$ \\
11886 & 0.15 & 0.54 & 24 & $1: 2.26$ \\
12336 & 0.70 & 0.53 & 18 & $1: 3.42$ \\
\hline
\end{tabular}

Notes. The table shows the flux cancellation rate, total amount of flux canceled, total percentage of peak unsigned AR flux canceled, and the ratio of the flux available to be built into the flux rope compared to the flux contained in the overlying arcade, i.e., the flux of the remaining bipole.

${ }^{a}$ Corrected values for total flux canceled, total percentage of peak AR flux and the ratio of the flux in the flux rope versus the overlying arcade are given for AR 11561.

include the fragmentation and advection of fragments across larger and larger areas by plasma flows. However, these flux fragments are captured in our method of flux measurement. In addition, Ohmic diffusion will cause flux to diffuse through the photosphere, due to the finite electrical resistance of the plasma. This diffusion process occurs on a timescale $t_{\mathrm{D}}$, which is given by $t_{\mathrm{D}}=L^{2} / \eta$, where $L$ is the length-scale and $\eta$ is the magnetic diffusivity. However, a sunspot of length-scale $3000 \mathrm{~km}$, using a value of Ohmic diffusion of $\eta=300 \mathrm{~m}^{2} \mathrm{~s}^{-1}$, gives a large diffusion timescale of the order 1000 years. By definition, flux cancellation as determined by our method can only be calculated during an AR's decay phase, when no new significant flux is emerging into the region and the overall flux value is reducing.

We also take into account that even though HMI produces high-quality data products, there are uncertainties and systematic errors present in the line-of-sight magnetic flux measurements. The selection criteria imposed when choosing ARs suitable for the study included that the regions had to emerge between $\pm 60^{\circ}$. This was to avoid the appearance of symmetric peaks, centered around $\sim 60^{\circ}$ with respect to central meridian due to the sensitivity of the HMI instrument being dependent upon longitude (Hoeksema et al. 2014; Couvidat et al. 2016). The increase in flux is caused by the increase in value by a few tens of percent of low to moderate flux densities between 250 and $750 \mathrm{G}$. However, this effect is still present before the AR reaches $60^{\circ}$. A recent study by Falconer et al. (2016) has used a sample of 272 large ARs to reduce the net projection error in parameters measured from deprojected $S D O / \mathrm{HMI}$ vector magnetograms. They remove the average projection error in an AR's total magnetic flux by assuming that the center-to-limb curve of the average of the absolute values of magnetic flux of a large number of ARs, which is normalized to the value at central meridian for each AR, gives the average fractional projection error at each radial distance from disk center. In this study we have not followed the method of Falconer et al. (2016) as we have only analyzed flux cancellation that occurs between $\sim \pm 45^{\circ}$. There are also sinusoidal oscillations with periods of 12 and $24 \mathrm{hr}$ in the evolution of total magnetic flux. This time-varying systematic error is mainly caused by the geosynchronous orbit of the $S D O$ spacecraft (Hoeksema et al. 2014).

Since we have selected isolated ARs and studied the flux cancellation that occurred during their decay phase, we are able to probe the characteristics of the ARs that produced eruptions from a low-altitude along their internal PILs and those that did not. Here we single out four ARs that produce internal PIL eruptions (ARs 11437, 11561, 11680 and 12382) and five ARs that produce only high-altitude eruptions (ARs 11446, 11808, 11881,11886 ) and analyze their decay phase. In Section 3.2 we described how both groups have roughly the same amount of total flux canceled although the ARs that produce high-altitude eruptions have, on average, a higher flux cancellation rate. These two groups of AR have a similar photospheric field evolution but markedly different outcomes in the evolution of the coronal field. These results lead to two questions. Why do ARs with a higher flux cancellation rate during their decay phase produce no eruptions from their internal PILs as the van Ballegooijen \& Martens (1989) flux rope model might suggest? What are the distinguishing features between these two groups of ARs?

These questions can be addressed by considering the ratio of canceled flux that is available to be built into the flux rope, versus the remaining flux in the overlying arcade. When more AR flux is canceled and built into a flux rope, there is less overlying field remaining in the AR to stabilize the structure. Previous observational flux cancellation studies have found a ratio of flux contained in the rope compared to the flux remaining in the overlying arcade of 1:0.65 (Green et al. 2011) and 1:0.9 (Yardley et al. 2016). Whereas, studies from a modeling perspective have yielded values between 1:1.5 and 1:1.9 (Bobra et al. 2008; Savcheva \& van Ballegooijen 2009; Savcheva et al. 2012). In this case we found that the ratio of flux canceled (i.e., the flux available to be built into the rope) compared to that in the overlying field at the time of the internal PIL event is: 1:1.29, 1:1.57, 1:0.03, 1:0.32 for ARs $11437,11561,11680$, and 12382 , respectively. We note that while for ARs 11437 and 11561 the ratio is very similar to previous results, for ARs 11680 and 12382 the flux contained in the overlying arcade is very small. This suggests that the assumption that flux injects an equal amount of flux into the rope as that canceled may not fully apply here.

This is due to the fact that the total flux canceled is equal to the amount of flux that is available to be built into the flux rope, and therefore represents an upper limit on the flux that has been built into the rope. However, the actual amount that builds into the rope is dependent upon the shear of the arcade and the length of the active section of the PIL where flux cancellation is taking place (Green et al. 2011). Both of these parameters can vary during the lifetime of an AR. An increase in the active section of the PIL and the shear of the arcade field can increase the chances of a loop being involved in a flux cancellation event at both of its ends. When this is the case, flux is canceled without contributing to the amount of flux in the rope. AR 11680 exhibits a strong increase in the length of the active section of the PIL, and AR 12382 shows a large increase in shear between the positive and negative polarities meaning that the amount of flux being built into rope may be overestimated. 
ARs that produce high-altitude events have a larger proportion of flux remaining in the overlying arcade compared to regions that produce internal PIL events. AR 11881 is an outlier in terms of the ratio for the high-altitude regions as it has a value of 1:0.94, which is within the range that produces internal PIL eruptions. However, when analyzing the AIA data in the time period following the end of our flux cancellation measurement we observe an internal PIL eruption that occurs on 2013 October 31 at around 01:50 UT. This is just over a day after our flux cancellation measurements ceased because the magnetic flux evolution could no longer be followed. There were no internal PIL events observed following the end of the flux cancellation measurement for the remaining ARs that produced high-altitude eruptions. Our results also show that the average shear angle of the ARs that produce internal PIL events is, on average, higher than that of the other event categories. These results suggest that flux cancellation within a sheared arcade may build a potentially eruptive configuration but that a successful eruption depends on the removal of sufficient overlying and stabilizing field.

In a recent study by Sterling et al. (2017) the evolution of a series of coronal jets that occurred at the periphery of the leading sunspot of AR 12259 were analyzed. They found that seven AR jets occurred during strong flux cancellation calculating an average flux cancellation rate of $1.5 \times 10^{19} \mathrm{Mx} \mathrm{h}^{-1}$ with an average of $\sim 5 \times 10^{18} \mathrm{Mx}$ canceled prior to each episode. The flux cancellation rates for the AR jets were found to be higher than the ARs in this study. This is not that surprising considering that the photospheric evolution of the jet-productive area is on the same size-scale as the ARs and the area is followed for a period of hours rather than days. On average, the total flux canceled in the ARs in this study was found to be 2 orders of magnitude larger than for the AR jets.

In this study we have focussed on the flux cancellation scenario of van Ballegooijen \& Martens (1989) and the role it plays in the productivity of eruptions in small and isolated bipolar ARs. This required an analysis of the relationship between flux cancellation, the evolution of the coronal magnetic field, and the eruption onset. We conclude that flux cancellation can be considered as a CME trigger if sufficient stabilizing field is removed from above the sheared core field. Other studies have investigated which nonpotentiality parameters are strong indicators that a CME will occur. For example, Bobra \& Ilonidis (2016) used features derived from SDO/HMI vector magnetograms to deduce whether ARs that produce M1 class flares or above will also produce a CME. They determined which features distinguish flaring ARs that produce CMEs from those that do not. The study found that the highest-performing features, which characterize the nonpotentiality of the magnetic field, are the mean horizontal gradient of the magnetic field and the twist parameter. A study by Tiwari et al. (2015) found that ARs with a larger nonpotentiality and total magnetic flux can produce both fast and slow CMEs, whereas smaller ARs with a more potential configuration can only produce slower CMEs. One key factor that plays a key role in CME productivity is the configuration of the overlying field (Török \& Kliem 2005). The gradient of the overlying field with height, for the ARs in our study, will be investigated in the future using nonlinear force-free modeling.

\section{Summary}

In this study, we analyzed a sample of 20 bipolar ARs over several days starting at the time of emergence, in order to investigate the importance of flux cancellation as a CME trigger. Following the model of van Ballegooijen \& Martens (1989), flux cancellation is the result of magnetic reconnection that is driven by the convergence and collision of loop footpoints in a sheared arcade. This process is able to build an eruptive structure, cut the tethers of the overlying field, and hence act as a CME trigger.

Flux cancellation was observed in all ARs with the exception of AR 11867, which remained in its emergence phase during the time period studied. In total, 24 eruptions were produced in 13 ARs. These eruptions were categorized into three types: low-altitude eruptions from the internal PIL (internal PIL events), low-altitude eruptions from an external PIL (external PIL events), and eruptions originating from high in the corona (high-altitude events). We found that the category of eruption produced is related to the evolutionary stage of the bipolar AR. For example, the majority of external PIL events occurred during the AR's emergence phase, when the growing bipole pushes into the surrounding, pre-existing magnetic field. This interaction can drive reconnection and flux cancellation, which may build an eruptive structure at the edge of the AR. The three external PIL events that occurred during the decay phase of their host AR appear to be related to the emergence of a small bipole close to the AR periphery. This forms an external PIL where cancellation can occur between opposite polarities. In contrast, internal PIL events only occurred during the bipole's decay phase when the bipole polarities have stopped separating and fragmented AR flux is being brought to the internal PIL via convective flows, driving reconnection and flux cancellation. High-altitude events occurred during both the emergence and decay phase of the ARs and could be the result of the destabilization of a pre-existing structure or the formation of a high-altitude structure formed during the evolution of the AR. We do not carry out an in-depth study of this category of eruption here, rather we leave this to a subsequent study. In summary, we found that no ARs produced both low- and highaltitude eruptions during the time they were studied and that low-altitude eruptions originating from external PILs were more common than those from internal PILs.

Internal PIL and external PIL eruption-productive regions had, on average, lower flux cancellation rates than the ARs that produced high-altitude eruptions and regions that produced no eruptions at all. However, the regions that produced low- or high-altitude eruptions exhibited a similar amount of total flux canceled, despite the difference in cancellation rates. Therefore, a high rate of flux cancellation and associated reconnection is not, on its own, a sufficient condition for eruption. For the four ARs that produced low-altitude events originating along the internal PIL we found that on average $36 \%$ of the peak AR flux had canceled prior to eruption. This is consistent with percentages found in previous studies (Green et al. 2011; Baker et al. 2012; Yardley et al. 2016) and is 12\% higher than the average value across noninternal PIL event ARs.

A secondary effect of the flux cancellation process is the reduction of AR flux that contributes to the magnetic field overlying and stabilizing the sheared core structure (that might contain a flux rope). If a flux rope has formed, and sufficient magnetic flux is transferred from the overlying arcade to this rope, an eruption may be produced. The quantity of flux 
canceled, which is equal to the amount available to be built into the flux rope, compared to the flux of the remaining overlying field was found to be in the range 1:0.03-1:1.57 and 1:2.26-1:3.42 for ARs that produced low-altitude internal PIL and high-altitude events, respectively. The ratio for AR 11881 has been omitted from consideration here because this region produced a low-altitude eruption originating from the internal PIL just over a day after the end of the flux cancellation measurement. Therefore, we find that a successful eruption originating from a low-altitude at the internal PIL depends upon the removal of a significant amount of the overlying field, which otherwise acts to stabilize the flux rope.

The nonpotentiality (or shear) of the arcade field is a key aspect of the van Ballegooijen \& Martens (1989) flux rope model. The shear angle measured at the time of the peak AR flux is, on average, $14^{\circ}$ higher for the regions that produced low-altitude internal PIL events compared to the regions that produced eruptions at high-altitude. An overall convergence of the bipole is important for the gradient of the coronal field that acts to stabilize any flux rope formed. We find that $75 \%$ of the ARs that produced low-altitude eruptions along their internal PIL showed a combination of bipole convergence, shear, and flux cancellation. Only $34 \%$ of the ARs that do not produce internal PIL eruptions show this combination.

In summary, we have conducted the first extensive study of eruptive activity in a sample of 20 small bipolar ARs taken from the $S D O / \mathrm{HMI}$ era in order to probe the role of flux cancellation as a CME trigger. The results of this study led to the conclusion that although flux cancellation plays a key role it is not sufficient, by itself, in the production of low-altitude eruptions. A combination of ongoing flux cancellation in a sheared arcade, which is consistent with the flux rope model of van Ballegooijen \& Martens (1989), can build a pre-eruptive configuration but a successful eruption depends upon the removal of sufficient overlying field that would otherwise stabilize the underlying flux rope. In this study the cancellation of more than $\sim 30 \%$ of the peak AR flux value appeared to be sufficient. In addition, the eruptions appear to be aided by the convergence of the bipole polarities.

The authors are grateful to the SDO/HMI and AIA consortia for the data, and also JHelioviewer (http://jhelioviewer.org/), which was used for browsing data. This research also makes use of SunPy (SunPy Community et al. 2015) (http://sunpy. org/), an open-source and free community-developed solar data analysis package written in Python. We are also grateful to Sally Dacie who helped with code development. S.L.Y. acknowledges STFC for support via PhD studentship and the Consolidated Grant SMC1 YST025. S.L.Y and L.M.G. are part of an International Space Science Institute (ISSI) team entitled "Decoding the Pre-Eruptive Magnetic Configurations of Coronal Mass Ejections." We are thankful to ISSI for hosting us, Spiros Patsourakos and Angelos Vourlidas for leading the team, and the rest of the group for valuable discussions. L.M.G. acknowledges support through a Royal Society University Research Fellowship. L.v.D.G. is partially funded under
STFC consolidated grant number ST/N000722/1. L.v.D.G. also acknowledges the Hungarian Research grant OTKA K-109276. L.M.G. and L.v.D.G. acknowledge funding under Leverhulme Trust Research Project Grant 2014-051. D.H.M. would like to thank both the STFC and Levehulme trust for financial support.

Software: JHelioviewer (http://jhelioviewer.org/), SunPy (SunPy Community et al. 2015).

\section{ORCID iDs}

S. L. Yardley (D) https://orcid.org/0000-0003-2802-4381

L. M. Green (iD https://orcid.org/0000-0002-0053-4876

D. H. Mackay (iD https://orcid.org/0000-0001-6065-8531

\section{References}

Amari, T., Luciani, J. F., Aly, J. J., Mikic, Z., \& Linker, J. 2003, ApJ, 585,1073

Aulanier, G., Török, T., Démoulin, P., \& DeLuca, E. E. 2010, ApJ, 708, 314 Baker, D., van Driel-Gesztelyi, L., \& Green, L. M. 2012, SoPh, 276, 219

Bernasconi, P. N., Rust, D. M., Georgoulis, M. K., \& Labonte, B. J. 2002, SoPh, 209, 119

Bobra, M. G., \& Ilonidis, S. 2016, ApJ, 821, 127

Bobra, M. G., van Ballegooijen, A. A., \& DeLuca, E. E. 2008, ApJ, 672, 1209

Chae, J., Moon, Y.-J., \& Pevtsov, A. A. 2004, ApJL, 602, L65

Couvidat, S., Schou, J., Hoeksema, J. T., et al. 2016, SoPh, 291, 1887

Dacie, S., Démoulin, P., van Driel-Gesztelyi, L., et al. 2016, A\&A, 596, A69

Eastwood, J. P., Biffis, E., Hapgood, M. A., et al. 2017, Risk Analysis, 37, 206

Falconer, D. A., Tiwari, S. K., Moore, R. L., \& Khazanov, I. 2016, ApJL, 833, L31

Forbes, T. G. 2000, JGR, 105, 23153

Forbes, T. G., \& Isenberg, P. A. 1991, ApJ, 373, 294

Gosling, J. T. 1993, JGR, 98, 18937

Green, L. M., Kliem, B., \& Wallace, A. J. 2011, A\&A, 526, A2

Green, L. M., Török, T., Vršnak, B., Manchester, W., \& Veronig, A. 2018, SSRv, 214, 46

Harvey, K. L., Jones, H. P., Schrijver, C. J., \& Penn, M. J. 1999, SoPh, 190, 35 Hoeksema, J. T., Liu, Y., Hayashi, K., et al. 2014, SoPh, 289, 3483

Kliem, B., Lin, J., Forbes, T. G., Priest, E. R., \& Török, T. 2014, ApJ, 789, 46 Kliem, B., \& Török, T. 2006, PhRvL, 96, 255002

Lemen, J. R., Title, A. M., Akin, D. J., et al. 2012, SoPh, 275, 17

Lin, J., \& Forbes, T. G. 2000, JGR, 105, 2375

Mackay, D. H., \& van Ballegooijen, A. A. 2006a, ApJ, 641, 577

Mackay, D. H., \& van Ballegooijen, A. A. 2006b, ApJ, 642, 1193

Martin, S. F., Livi, S. H. B., \& Wang, J. 1985, AuJPh, 38, 929

Moore, R. L., Sterling, A. C., Hudson, H. S., \& Lemen, J. R. 2001, ApJ, 552,833

Pesnell, W. D., Thompson, B. J., \& Chamberlin, P. C. 2012, SoPh, 275, 3

Savcheva, A., \& van Ballegooijen, A. 2009, ApJ, 703, 1766

Savcheva, A. S., Green, L. M., van Ballegooijen, A. A., \& DeLuca, E. E. 2012, ApJ, 759, 105

Schou, J., Scherrer, P. H., Bush, R. I., et al. 2012, SoPh, 275, 229

Sterling, A. C., Moore, R. L., Falconer, D. A., Panesar, N. K., \& Martinez, F. 2017, ApJ, 844, 28

SunPy Community, Mumford, S. J., Christe, S., et al. 2015, CS\&D, 8, 014009

Tiwari, S. K., Falconer, D. A., Moore, R. L., et al. 2015, GeoRL, 42, 5702

Török, T., \& Kliem, B. 2005, ApJL, 630, L97

van Ballegooijen, A. A., \& Martens, P. C. H. 1989, ApJ, 343, 971

van Driel-Gesztelyi, L., Malherbe, J.-M., \& Démoulin, P. 2000, A\&A, 364,845

Yang, S., Zhang, J., \& Borrero, J. M. 2009, ApJ, 703, 1012

Yardley, S. L., Green, L. M., Williams, D. R., et al. 2016, ApJ, 827, 151

Zwaan, C. 1987, A\&A, 25, 83 